\title{
Effect of plantar subcutaneous administration of bergamot essential oil and linalool on formalin-induced nociceptive behavior in mice
}

\author{
Soh Katsuyama ${ }^{1}$, Akira Otowa ${ }^{1}$, Satomi Kamio ${ }^{1}$, Kazuma Sato ${ }^{1}$, Tomomi Yagi ${ }^{1}$, Yukinaga Kishikawa ${ }^{1}$, \\ Takaaki Komatsu ${ }^{2}$, Giacinto Bagetta ${ }^{3}$, Tsukasa Sakurada ${ }^{2}$, and Hitoshi Nakamura ${ }^{1}$ \\ ${ }^{1}$ Department of Clinical Pharmaceutics, Tohoku Pharmaceutical University, 4-4-1 Komatsushima, Aoba-ku, Sendai 981-8558, Japan; \\ ${ }^{2}$ Department of Pharmacology, Daiichi College of Pharmaceutical Sciences, 22-1 Tamagawa-cho, Minami-ku, Fukuoka 815-8511, Japan; \\ and ${ }^{3}$ Department of Pharmacobiology and University Consortium for Adaptive Disorders and Headache, Section of Neuropharmacology \\ of Normal and Pathological Neuronal Plasticity, University of Calabria, 87036 Arcavacata di Rende, Italy
}

(Received 14 October 2014; and accepted 14 November 2014)

\begin{abstract}
This study investigated the effect of bergamot essential oil (BEO) or linalool, a major volatile component of BEO, on the nociceptive response to formalin. Plantar subcutaneous injection of $\mathrm{BEO}$ or linalool into the ipsilateral hindpaw reduced both the first and late phases of the formalininduced licking and biting responses in mice. Plantar subcutaneous injection of BEO or linalool into the contralateral hindpaw did not yield an antinociceptive effect, suggesting that the antinociceptive effect of BEO or linalool in the formalin test occurred peripherally. Intraperitoneal and plantar subcutaneous injection pretreatment with naloxone hydrochloride, an opioid receptor antagonist, significantly attenuated both BEO- and linalool-induced antinociception. Pretreatment with naloxone methiodide, a peripherally acting opioid receptor antagonists, also significantly antagonized the antinociceptive effects of BEO and linalool. Our results provide evidence for the involvement of peripheral opioids in antinociception induced by BEO and linalool. These results suggest that activation of peripheral opioid receptors may play an important role in reducing formalin-induced nociception.
\end{abstract}

The essential oil of bergamot (BEO; Citrus bergam$i a$ Risso) is one of the most commonly used essential oils and is familiar to most of the general public. BEO is obtained by cold pressing of the epicarp and part of the mesocarp of the fresh bergamot fruit. BEO consists of a volatile (93-96\%) and a nonvolatile fraction $(4-7 \%)$; the former contains monoterpene and sesquiterpene hydrocarbons and oxygenated derivatives such as linalool and linalyl acetate, while the latter fraction contains waxes, polymethoxylated flavones, coumarins, and pso-

Address correspondence to: Hitoshi Nakamura Department of Clinical Pharmaceutics, Tohoku Pharmaceutical University, 4-4-1 Komatsushima, Aoba-ku, Sendai 981-8558, Japan

Tel: +81-22-727-0211, Fax: +81-22-727-0149

E-mail: nakamura@tohoku-pharm.ac.jp ralens such as bergamottin and bergapten $(6,14)$. BEO has been reported to minimize symptoms of stress-induced anxiety and mild mood disorders, as well as cancer pain, however the mechanistic basis for its use in such applications awaits discovery (1).

A previous in vitro study showed that BEO reduced neuronal damage caused by excitotoxic stimuli (5), and significantly increased the extracellular levels of the inhibitory amino acid neurotransmitter gamma-aminobutyric acid (GABA) in rat hippocampus (15). Linalool is a monoterpene compound and is the main volatile component of the essential oils of various plants, including BEO. It has previously been reported that linalool administration produced antibacterial, anticonvulsant, and anti-inflammatory effects, as well as showing antinociceptive activity in several behavioral assays $(2-4,16-20)$. Furthermore, linalool can significantly reduce both mor- 
phine tolerance and dependence (9). However, the antinociceptive efficacy of plantar subcutaneous injection of BEO and linalool on the formalin-induced nociceptive response is unknown.

The formalin test is an experimental model by which to assess an animal's response to moderate, continuous pain generated by tissue damage. When injected into the hindpaw of mice and rats, formalin induces characteristic biphasic licking and biting behaviors in response to continuous noxious stimuli originating from the changes occurring at the site of injection. The behavior consists of a first phase, occurring about 5 min after the injection of formalin, and then after a quiescent period, a second phase occurring from 10 to $30 \mathrm{~min}$ after injection. The first phase occurs in response to direct stimulation of the sensory nerve endings by formalin and indicates acute pain, while the second phase occurs in response to the ensuing inflammation and indicates persistent pain $(10,25,26)$.

The main aim of the present study was to investigate whether plantar subcutaneous injection of BEO or linalool would produce antinociception in the context of formalin-induced nociception in mice. In addition, this study sought to assess the involvement of the peripheral opioid system in the antinociceptive effects of BEO and linalool.

\section{MATERIALS AND METHODS}

Animals. Male ddY-strain mice (Japan SLC, Hamamatsu, Japan) weighing an average of $23-25 \mathrm{~g}$ at the time of testing were used in these experiments. The mice were individually housed in a colony maintained in a controlled environment $(12 \mathrm{~h}$ light/ dark cycle, room temperature $23^{\circ} \mathrm{C}, 50-60 \%$ relative humidity). The animals had unlimited access to food pellets and water. All behavioral experiments took place during the light period between the hours of $10: 00$ and 16:00 in a quiet room. The animals belonging to the various treatment groups $(n=10$ each group) were tested in randomized order. All experiments followed the Guidelines on Ethical Standards for Investigation of Experimental Pain in Animals (29). Additionally, the study was approved by the Committees of Animal Care and Use of Tohoku Pharmaceutical University.

Materials. BEO was kindly provided by the Simone Gatto company (San Pier Niceto, Messina, Italy) together with a certificate of analysis performed by the Stazione Sperimentale per le Industrie delle Essenze e dei Derivati dagli Agrumi (Reggio Calabria,
Italy). The composition of the essential oil of bergamot used here has been previously reported by Corasaniti et al. (5). Briefly, BEO contained 0.38\% D-limonene, $70.26 \%$ linalyl acetate, $18.95 \%$ linalool, $0.62 \% \gamma$-terpinene, and $0.03 \% \beta$-pinene. The following drugs and chemicals were used: $( \pm)$ linalool and formalin (Nacalai Tesque, Kyoto, Japan), naloxone hydrochloride and naloxone methiodide (Sigma Chemical Co., St. Louis, MO). BEO and linalool were diluted in jojoba wax (Simmondsia chinensis) (K.S.A. International Co. Ltd., Kanagawa, Japan) to reach total amounts of $1.25-10 \mu \mathrm{g}(11,23)$. Jojoba wax alone had no effect on formalin-induced nociception. Formalin, naloxone hydrochloride, and naloxone methiodide were dissolved in physiological saline $(0.9 \% \mathrm{wt} / \mathrm{vol})$.

Formalin test. In the formalin test, mice were placed into a transparent cage $(22.0 \mathrm{~cm} \times 15.0 \mathrm{~cm} \times 12.5 \mathrm{~cm}$ high) which also served as an observation chamber and were allowed to adapt to their environment for $1 \mathrm{~h}$ before testing. After this period, plantar subcutaneous injection of $20 \mu \mathrm{L}$ formalin ( $2 \%$ in saline) using a microsyringe with 26-gauge needle. Each mouse was immediately returned to the observation chamber after injection. The recording of the first response (first phase) started immediately and lasted for $10 \mathrm{~min}(0-10 \mathrm{~min})$. The recording of the late response (late phase) started $10 \mathrm{~min}$ after formalin injection and lasted for $20 \mathrm{~min}(10-30 \mathrm{~min})$. In both phases, licking and biting of the injected hindpaw were defined as a nociceptive response and the total time (s) of the response was measured with a handheld stop-watch.

Experimental protocol. BEO and ( \pm ) linalool were injected plantar surface of the right hindpaw $10 \mathrm{~min}$ before local injection of formalin (23). The opioid receptor antagonists, naloxone hydrochloride and its quaternary form, naloxone methiodide, were preinjected intraperitoneally (i.p.) $30 \mathrm{~min}$ before plantar subcutaneous injection of BEO and linalool. Naloxone hydrochloride was injected into the hindpaw 15 min before plantar subcutaneous injection of BEO and linalool. Naloxone methiodide is thought not to cross the blood-brain barrier and is used as a research tool to peripheral sites of action for drugs acting on opioid receptors (13). The effect of BEO and linalool, administered the plantar surface of the contralateral (left hindpaw) or ipsilateral paws, was also studied. In all experiments the observer was unaware of the treatment. Plantar subcutaneous and i.p. injections were given in a volume of $20 \mu \mathrm{L} /$ site and 
$0.1 \mathrm{~mL} / 10 \mathrm{~g}$ of body weight, respectively.

Data analysis. Data are presented as means \pm S.E.M. of 10 mice per experimental group. Statistical differences between groups were assessed with a oneway or two-way ANOVA followed by Dunnett's or Bonferroni's test. The level of statistical significance was set at $5 \%(P<0.05)$ in all experiments.

\section{RESULTS}

Antinociceptive effects of plantar subcutaneous administration of BEO and linalool in the formalin test Plantar subcutaneous injection of $2 \%$ formalin caused biphasic nociceptive behavior consisting of licking and biting. The first phase (phase I) occurred within the first 10 min following injection and disappeared almost completely by 5-10 min (Fig. 1). Subsequently, the nociceptive response reoccurred 10-30 min after the injection of formalin (late phase; phase II) (Fig. 1). To evaluate their antinociceptive effects, BEO, linalool, or jojoba wax was injected $10 \mathrm{~min}$ before formalin at the same site. The nociceptive behavioral response (phases I and II) to plantar subcutaneous formalin injection after the jojoba wax control was not statistically when compared with that in the group which was not pre-injected (Fig. 1 and 2A-D). In contrast, plantar subcutaneous injection of BEO $(10 \mu \mathrm{g})$ and linalool $(2.5 \mu \mathrm{g}$ and $5 \mu \mathrm{g})$ significantly inhibited the nociceptive behavioral response to formalin (Fig. 1 and 2A-D).

Effects of BEO and linalool injected into the hindpaw contralateral to the formalin injection

To ensure that the effects of plantar subcutaneous injection of BEO and linalool were peripheral and not due to systemic diffusion of each compound, BEO $(10 \mu \mathrm{g})$ or linalool $(5 \mu \mathrm{g})$ was injected into the hindpaw contralateral to the formalin injection. Formalin-induced nociceptive behavior (phases I and II) following injection of either $\mathrm{BEO}$ or linalool into the contralateral hindpaw was not statistically different when compared with the jojoba wax-treated control group (Fig. 2A-D).

Effects of naloxone hydrochloride on antinociception induced by BEO and linalool

To determine if the antinociceptive effects of BEO and linalool were mediated by opioid systems, animals were pretreated i.p. with the opioid receptor antagonist naloxone hydrochloride, $30 \mathrm{~min}$ before injection of BEO $(10 \mu \mathrm{g})$ or linalool $(5 \mu \mathrm{g})$, which was performed $10 \mathrm{~min}$ prior to formalin injection.

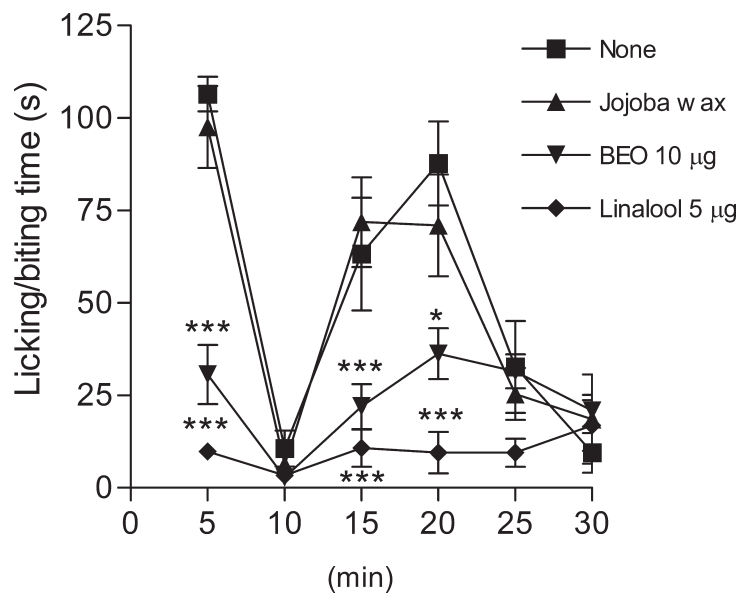

Fig. 1 Time course of nociceptive behavior induced by formalin $(2 \%, 20 \mu \mathrm{L})$ in mice treated with none, jojoba wax, bergamot essential oil (BEO) $(10 \mu \mathrm{g})$ and linalool $(5 \mu \mathrm{g})$ $10 \mathrm{~min}$ prior to $2 \%$ formalin. Values represent the mean $\pm \mathrm{S}$. E.M. for 10 mice in each group. Statistical differences between the groups were assessed by two-way ANOVA followed by Bonferroni's test. ${ }^{\star} P<0.05,{ }^{\star \star \star} P<0.001$ compared with the jojoba wax control.

Naloxone hydrochloride (16 mg/kg, i.p.) significantly reversed the inhibitory effects of BEO or linalool on the formalin-induced behavioral response (Fig. 3AD). In further experiments, naloxone hydrochloride was injected directly into the same site on the hindpaw before plantar subcutaneous injection of BEO or linalool. Plantar subcutaneous injection pretreatment with naloxone hydrochloride (16 or $32 \mu \mathrm{g}$ ) also significantly and dose-dependently antagonized the antinociceptive effects of BEO or linalool (Fig. 4A-D).

Effect of naloxone methiodide on antinociception induced by BEO or linalool

To investigate the involvement of peripheral opioid receptors, we examined the effect of peripherally acting opioid antagonist naloxone methiodide on the $\mathrm{BEO}$ or linalool action. Pretreatment with naloxone methiodide (4 or $8 \mathrm{mg} / \mathrm{kg}$, i.p.) resulted in a significantly and dose-dependently antagonized antinociceptive effects of BEO or linalool (Fig. 5A-D).

\section{DISCUSSION}

The effects of the essential oil, BEO and of its main oxygenated monoterpene linalool were investigated in a mouse formalin pain model. Plantar subcutaneous injection of either BEO or linalool reduced behavioral signs of formalin-induced nociception in a dose-dependent manner. Neither BEO nor linalool injected into the contralateral paw was antinocicep- 

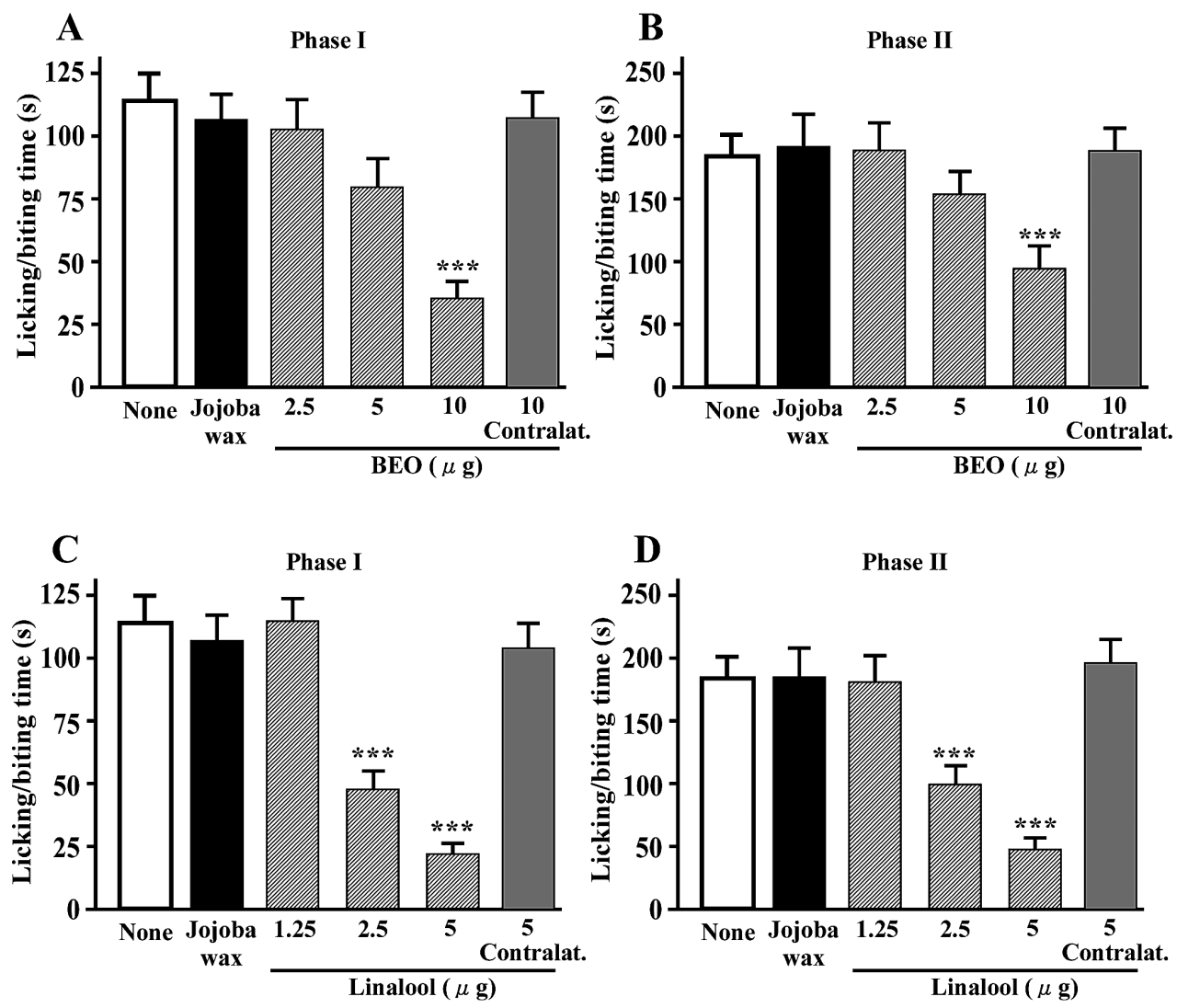

Fig. 2 Effects of bergamot essential oil (BEO) (A, B) or linalool (C, D) injected into the hindpaw, ipsilateral or contralateral (Contralat.) to the site of formalin injection, on formalin-induced biphasic nociceptive behavior in mice. Each compound was injected subcutaneously $10 \mathrm{~min}$ prior to $2 \%$ formalin. Nociceptive behavior in both the first $(0-10$ min, Phase I; A, C) and late (10-30 min, Phase II; B, D) phases was scored as the amount of time spent licking and biting the injected paw. Jojoba wax was used as a control and this failed to affect formalin-induced biphasic nociceptive behavior. Values represent the mean \pm S.E.M. for 10 mice in each group. Statistical differences between the groups were assessed by one-way ANOVA followed by Dunnett's test. ${ }^{* \star} P<0.001$ compared with the jojoba wax control.

tive at a dose which was sufficient to confer antinociception when injected into the ipsilateral paw. This finding suggests that BEO- and linalool-induced antinociception may be mediated peripheral. The present study further demonstrated that antinociception induced by plantar subcutaneous injection of BEO or linalool was antagonized by pretreatment with plantar subcutaneous administration of naloxone hydrochloride and the peripherally acting opioid receptor antagonist naloxone methiodide. These data suggest that plantar subcutaneous injection of BEO or linalool could produce antinociception that is, at least in part, mediated through peripheral opioid mechanisms.

The formalin model of nociception is useful to investigate both acute and continuous (tonic) nociception as there is a biphasic behavioral response (first phase and second phase) to formalin. The first phase reflects an acute, transient chemical stimulus and the second phase involves peripheral inflammation and central sensitization (10). The nociceptive behavioral response consists of licking and biting the injected paw $(21,24)$.

BEO protects against $N$-methyl-D-aspartate (NMDA)induced cell death by inducing the sustained phosphorylation of Akt kinase (5). Also, BEO significantly increased the extracellular levels of GABA in the hippocampus in freely moving rats (15). Linalool inhibited the biting response induced by intrathecal injection of IL-1 $\beta$ or TNF- $\alpha$ (3). Available evidence indicates that linalool could produce antinociception through interactions with opioid, muscarinic M2 or adenosine A1 receptors, or by modulating nitric oxide (NO) synthesis $(16,18-20)$. There is also evidence to suggest that linalool modulates glutamatergic neurotransmission via NMDA receptors $(2,7)$. Recently, we reported that plantar subcutaneous injection of $\mathrm{BEO}$ or linalool reduced nociceptive responses in 

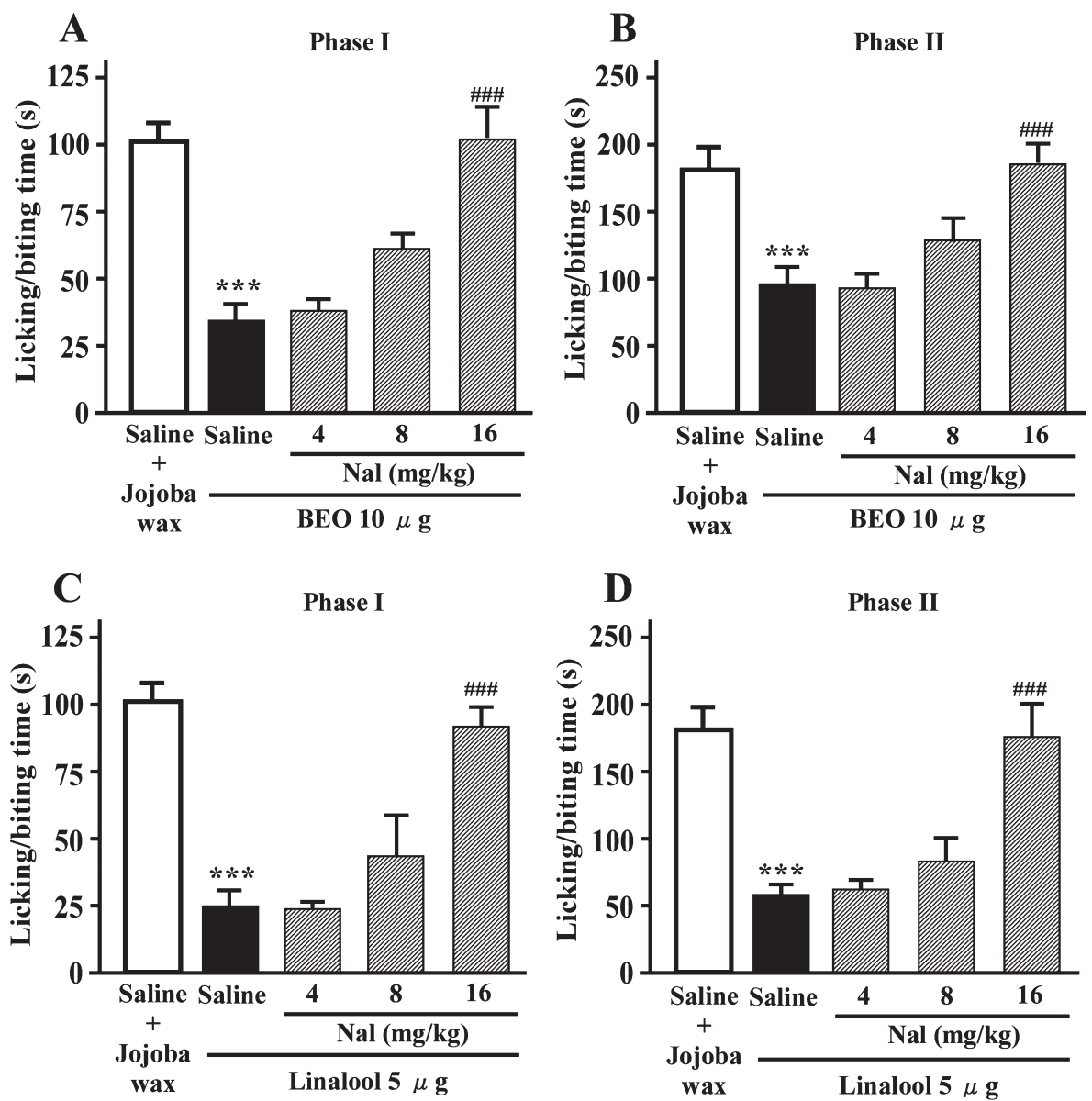

Fig. 3 Antagonism induced by i.p. injection of naloxone hydrochloride (Nal) of antinociception produced by bergamot essential oil (BEO) (A, B) or linalool (C, D). Nal was pre-injected i.p. 30 min before plantar subcutaneous injection of BEO or linalool. Values represent the mean \pm S.E.M. for 10 mice per group. Statistical differences between the groups were assessed with one-way ANOVA followed by Dunnett's test. ${ }^{* *} P<0.001$ compared with the saline (i.p.) + jojoba wax control.

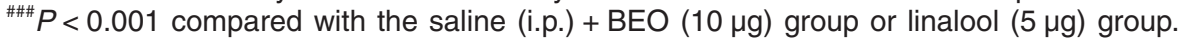

the acute pain models $(11,23)$, suggesting that BEO and linalool may be effective in reducing nociception linked to a range of paradigms.

The present data suggest that plantar subcutaneous injection of BEO or linalool significantly reduces formalin-induced nociception. Plantar subcutaneous injection of BEO or linalool into the contralateral hindpaw did not yield antinociceptive effects on formalin-induced nociception, strongly supporting a peripheral effect of BEO or linalool on cutaneous nociceptors. In order to assess the possible participation of either opioid receptors or opioid peptides in plantar subcutaneous injection of linalool-induced antinociception, the effect of opioid receptor antagonists on the antinociceptive activity of linalool was tested. The antinociceptive effect produced by linalool was reversed by plantar subcutaneous injection pretreatment with naloxone hydrochloride. Similar results were obtained with respect to plantar subcutaneous injection of BEO-induced antinociception. These results suggest that both BEO- and linaloolinduced antinociception may be mediated through opioid receptors in the periphery. This hypothesis is supported by the observation that pretreatment with the peripherally acting opioid receptor antagonist naloxone methiodide significantly antagonized the antinociception conferred by plantar subcutaneous injection of BEO or linalool. Naloxone methiodide may interact with opioid receptors associated with peripheral nerve endings.

It is conceivable that plantar subcutaneous injection of BEO and linalool might cause the peripheral release of endogenous opioid peptides and that these might be responsible for the peripherally mediated antinociceptive effect. Keratinocytes are known to synthesize and secrete the opioid peptide $\beta$-endorphin 

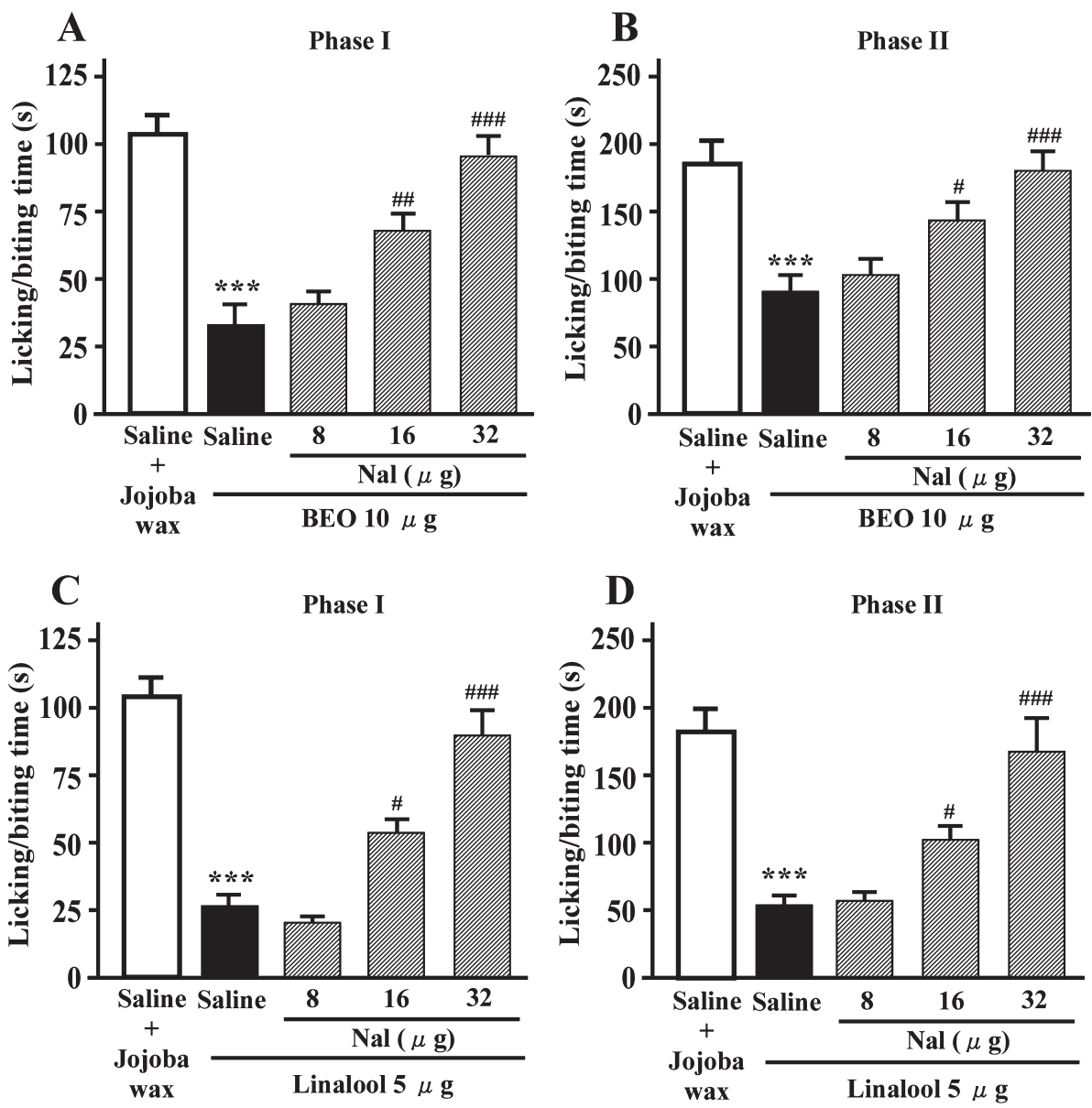

Fig. 4 Antagonism induced by plantar subcutaneous injection of naloxone hydrochloride (Nal) of antinociception produced by bergamot essential oil (BEO) (A, B) or linalool (C, D). Nal was pre-injected into the hindpaw 15 min before plantar subcutaneous injection of BEO or linalool. Values represent the mean \pm S.E.M. for 10 mice per group. Statistical differences between the groups were assessed with one-way ANOVA followed by Dunnett's test. ${ }^{* \star *} P<0.001$ compared with the saline (plantar subcutaneous injection) + jojoba wax control. ${ }^{\#} P<0.05,{ }^{\# \#} P<0.01$, ${ }^{\# \# \#} P<0.001$ compared with the saline (plantar subcutaneous injection) + BEO $(10 \mu \mathrm{g})$ group or linalool $(5 \mu \mathrm{g})$ group.

$(27,28)$. It is worth noting that proopiomelanocortin, the precursor of a variety of neuropeptides including $\beta$-endorphin $(8,12)$, is constitutively expressed by keratinocytes that are abundant in the skin.

The most abundant component of BEO is the monoterpene alcohol linalool and its corresponding ester (linalyl acetate). Linalool and linalyl acetate have been shown to possess anti-inflammatory and antinociceptive properties (23). Previously we showed that linalool was much more potent than either linalyl acetate or BEO in inhibiting the nociceptive response to plantar subcutaneous injection of capsaicin (23). Indeed, injection of the hindpaw with essential oil of sweet orange (Citrus sinensis), which is known to contain extremely small amounts of linalool and linalyl acetate, lacked significant antinociceptive ac- tivity in the capsaicin test (22). It seems likely that BEO-induced antinociception and antiallodynia may be dependent on the amount of linalool and/or linalyl acetate present in the oil. In agreement with this hypothesis, the present study showed that linalool possessed more potent antinociceptive activity than $\mathrm{BEO}$ in the formalin test.

In conclusion, plantar subcutaneous injection of BEO and linalool each reduced the nociceptive response as assayed by the formalin test. Furthermore, our data suggest that the antinociceptive effects of BEO and linalool were antagonized by the plantar subcutaneous injection of naloxone hydrochloride and i.p. naloxone methiodide, which act as antagonists at predominantly peripheral opioid receptors. Linalool had more potent antinociceptive activity than BEO in the formalin test. The present results 

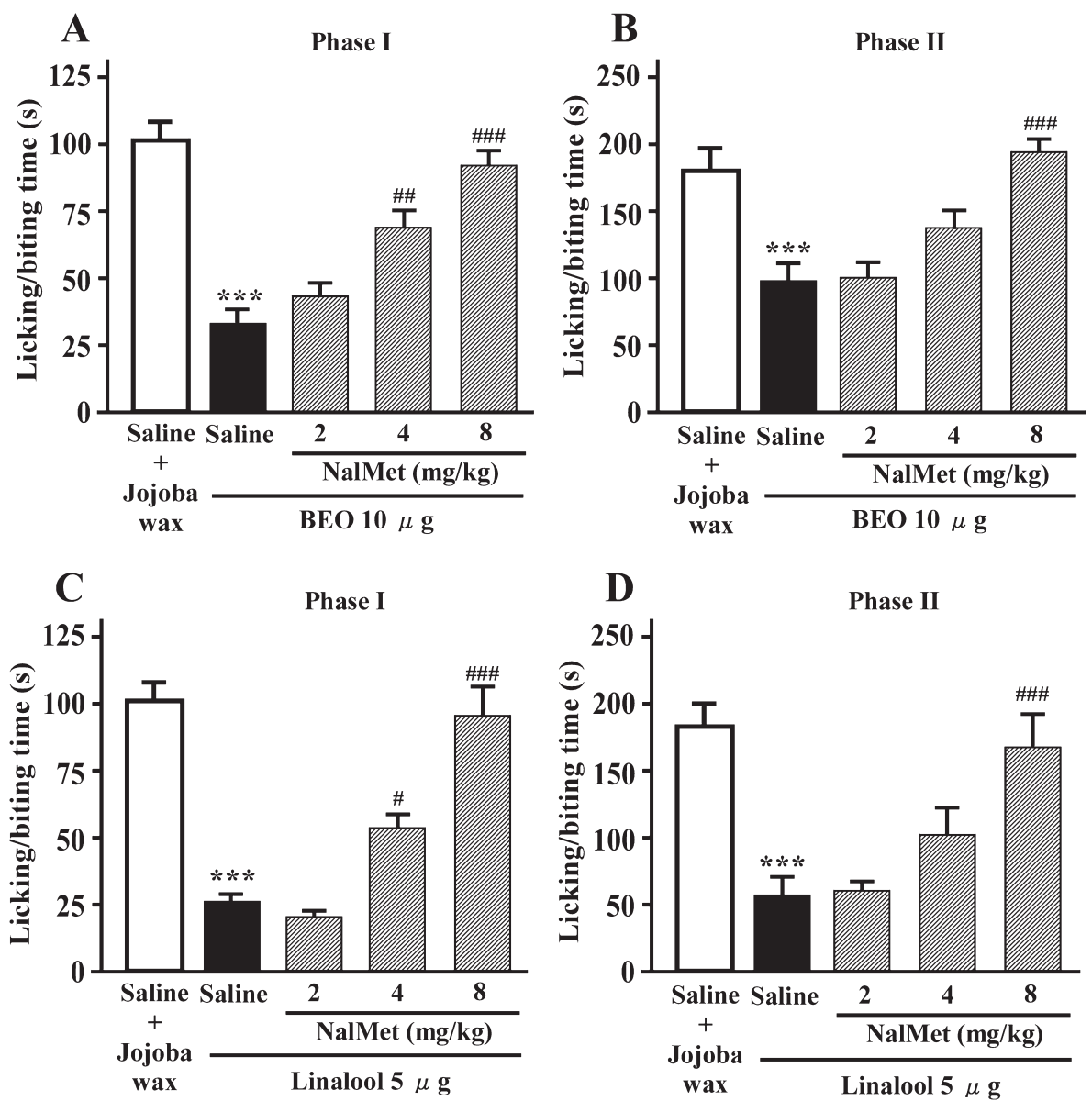

Fig. 5 Antagonism induced by i.p. injection of naloxone methiodide (NalMet) of antinociception produced by bergamot essential oil (BEO) (A, B) or linalool (C, D). NalMet was pre-injected i.p. 30 min before plantar subcutaneous injection of BEO or linalool. Values represent the mean \pm S.E.M. for 10 mice per group. Statistical differences between the groups were assessed with one-way ANOVA followed by Dunnett's test. ${ }^{\star \star \star} P<0.001$ compared with the saline (i.p.) + jojoba wax control. ${ }^{\#} P<0.05,{ }^{\# \#} P<0.01,{ }^{\# \# \#} P<0.001$ compared with the saline (i.p.) + BEO $(10 \mu \mathrm{g})$ group or linalool $(5 \mu \mathrm{g})$ group.

indicate that the use of either BEO or linalool may be a promising therapeutic approach to managing formalin-induced pain.

\section{Acknowledgement}

This study was supported by a NISHINOMIYA Basic Research Fund (Japan).

\section{REFERENCES}

1. Bagetta G, Morrone LA, Rombolà L, Amantea D, Russo R, Berliocchi L, Sakurada S, Sakurada T, Rotiroti D and Corasaniti MT (2010) Neuropharmacology of the essential oil of bergamot. Fitoterapia 81, 453-461.

2. Batista PA, Werner MF, Oliveira EC, Burgos L, Pereira P, Brum LF and Santos AR (2008) Evidence for the involvement of ionotropic glutamatergic receptors on the antinociceptive effect of (-)-linalool in mice. Neurosci Lett 440, 299-303.
3. Batista PA, Werner MF, Oliveira EC, Burgos L, Pereira P, Brum LF, Story GM and Santos AR (2010) The antinociceptive effect of (-)-linalool in models of chronic inflammatory and neuropathic hypersensitivity in mice. J Pain 11, 12221229.

4. Berliocchi L, Russo R, Levato A, Fratto V, Bagetta G, Sakurada S, Sakurada T, Mercuri NB and Corasaniti MT (2009) (-)-Linalool attenuates allodynia in neuropathic pain induced by spinal nerve ligation in $\mathrm{c} 57 / \mathrm{bl} 16$ mice. Int Rev Neurobiol 85, 221-235.

5. Corasaniti MT, Maiuolo J, Maida S, Fratto V, Navarra M, Russo R, Amantea D, Morrone LA and Bagetta G (2007) Cell signaling pathways in the mechanisms of neuroprotection afforded by bergamot essential oil against NMDAinduced cell death in vitro. Br J Pharmacol 151, 518-529.

6. Dugo P, Mondello L, Dugo L, Gugo L, Stancanelli R and Dugo P (2000) LC-MS for the identification of oxygen heterocyclic compounds in citrus essential oils. J Pharm Biomed Anal 24, 147-154.

7. Elisabetsky E, Silva Brum LF and Souza DO (1999) Anticonvulsant properties of linalool in glutamate-related seizure 
models. Phytomedicine 6, 107-113.

8. Galiegue S, Mary S, Marchand J, Dussossoy D, Carriere D, Carayon P, Bouaboula M, Shire D, Le Fur G and Casellas P (1995) Expression of central and peripheral cannabinoid receptors in human immune tissues and leukocyte subpopulations. Eur J Biochem 232, 54-61.

9. Hosseinzadeh $\mathrm{H}$, Imenshahidi $\mathbf{M}$, Hosseini $M$ and Razavi BM (2012) Effect of linalool on morphine tolerance and dependence in mice. Phytother Res 26, 1399-1404.

10. Hunskaar S and Hole K (1987) The formalin test in mice: dissociation between inflammatory and non-inflammatory pain. Pain 30, 103-114.

11. Katsuyama S, Kuwahata H, Yagi T, Kishikawa Y, Komatsu T, Sakurada T and Nakamura H (2012) Intraplantar injection of linalool reduces paclitaxel-induced acute pain in mice. Biomed Res (Tokyo) 33, 175-181.

12. Khodorova A, Navarro B, Jouaville LS, Murphy JE, Rice FL, Mazurkiewicz JE, Long-Woodward D, Stoffel M, Strichartz GR, Yukhananov R and Davar G (2003) Endothelin-B receptor activation triggers an endogenous analgesic cascade at sites of peripheral injury. Nat Med 9, 1055-1061.

13. Lewanowitsch $T$ and Irvine RJ (2002) Naloxone methiodide reverses opioid-induced respiratory depression and analgesia without withdrawal. Eur J Pharmacol 445, 61-67.

14. Mondello L, Stagno D'Alcontres I, Del Duce R and Crispo F (1993) On the genuineness of citrus essential oils. Part XL. The composition of the coumarins and psoralens of Calabria bergamot essential oil (Citrus bergamia Rissso). Flavour Fragr J 8, 17-24.

15. Morrone LA, Rombolà L, Pelle C, Corasaniti MT, Zappettini S, Paudice P, Bonanno G and Bagetta G (2007) The essential oil of bergamot enhances the levels of amino acid neurotransmitters in the hippocampus of rat: implication of monoterpene hydrocarbons. Pharmacol Res 55, 255-262.

16. Peana AT, D'Aquila PS, Chessa ML, Moretti MD, Serra G and Pippia P (2003) (-)-Linalool produces antinociception in two experimental models of pain. Eur J Pharmacol 460, 3741.

17. Peana AT, D’Aquila PS, Panin F, Serra G, Pippia P and Moretti MD (2002) Anti-inflammatory activity of linalool and linalyl acetate constituents of essential oils. Phytomedicine 9, 721-726.

18. Peana AT, De Montis MG, Nieddu E, Spano MT, D'Aquila PS and Pippia P (2004) Profile of spinal and supra-spinal an- tinociception of (-)-linalool. Eur J Pharmacol 485, 165-174.

19. Peana AT, Marzocco S, Popolo A and Pinto A (2006) (-)-Linalool inhibits in vitro NO formation: Probable involvement in the antinociceptive activity of this monoterpene compound. Life Sci 78, 719-723.

20. Peana AT, Rubattu P, Piga GG, Fumagalli S, Boatto G, Pippia P and De Montis MG (2006) Involvement of adenosine A1 and A2 receptors in (-)-linalool-induced antinociception. Life Sci 78, 2471-2474.

21. Sakurada T, Katsumata K, Yogo H, Tan-No K, Sakurada S, Ohba M and Kisara K (1995) The neurokinin-1 receptor antagonist, sendide, exhibits antinociceptive activity in the formalin test. Pain 60, 175-180.

22. Sakurada T, Kuwahata H, Katsuyama S, Komatsu T, Morrone LA, Corasaniti MT, Bagetta G and Sakurada S (2009) Intraplantar injection of bergamot essential oil into the mouse hindpaw: Effects on capsaicin-induced nociceptive behaviors. Int Rev Neurobiol 85, 237-248.

23. Sakurada T, Mizoguchi H, Kuwahata H, Katsuyama S, Komatsu T, Morrone LA, Corasaniti MT, Bagetta G and Sakurada S (2011) Intraplantar injection of bergamot essential oil induces peripheral antinociception mediated by opioid mechanism. Pharmacol Biochem Behav 97, 436-443.

24. Sato T, Sakurada S, Takahashi N, Sakurada T, Tan-No K, Wako K and Kisara K (1999) Contribution of spinal mu1opioid receptors to morphine-induced antinociception. Eur $J$ Pharmacol 369, 183-187.

25. Shields SD, Cavanaugh DJ, Lee H, Anderson DJ and Basbaum AI (2010) Pain behavior in the formalin test persists after ablation of the great majority of C-fiber nociceptors. Pain 151, 422-429.

26. Tjølsen A, Berge OG, Hunskaar S, Rosland JH and Hole K (1992) The formalin test: an evaluation of the method. Pain 51, 5-17.

27. Wintzen M, Yaar M, Burbach JP and Gilchrest BA (1996) Propiomelanocortin gene product regulation in keratinocytes. J Invest Dermatol 106, 673-678.

28. Zanello SB, Jackson DM and Holick MF (1999) An immunocytochemical approach to the study of beta-endorphin production in human keratinocytes using confocal microscopy. Ann N Y Acad Sci 885, 85-99.

29. Zimmermann M (1983) Ethical guidelines for investigation of experimental pain in conscious animals. Pain 16,109-110. 